\title{
«Rebelle ou brigand ? " Le baron de Saint-Christol (1748-1819) vu par lui-même et par ses juges
}

«Rebel or Brigand? " The Baron de Saint-Christol (1748-1819) in his own words and by his own judges

\section{Valérie Sottocasa}

\section{(2) OpenEdition}

\section{Journals}

\section{Édition électronique}

URL : https://journals.openedition.org/ahrf/12885

DOI : $10.4000 / a h r f .12885$

ISSN : 1952-403X

Éditeur :

Armand Colin, Société des études robespierristes

Édition imprimée

Date de publication : 1 septembre 2013

Pagination : 189-208

ISBN : 9782200928261

ISSN : 0003-4436

\section{Référence électronique}

Valérie Sottocasa, « «Rebelle ou brigand ? » Le baron de Saint-Christol (1748-1819) vu par lui-même et par ses juges », Annales historiques de la Révolution française [En ligne], 373 | juillet-septembre 2013, mis en ligne le 01 septembre 2016, consulté le 01 juillet 2021. URL : http://journals.openedition.org/ ahrf/12885; DOI : https://doi.org/10.4000/ahrf.12885 


\title{
«REBELLE OU BRIGAND? » LE BARON DE SAINT-CHRISTOL (1748-1819) VU PAR LUI-MÊME ET PAR SES JUGES
}

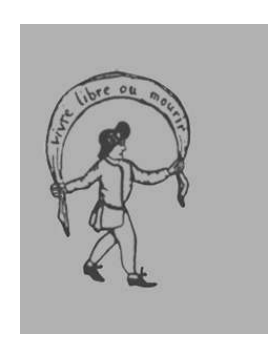

\author{
Valérie SOTTOCASA
}

\begin{abstract}
Les départements du Midi ont été agités par des troubles politiques précoces et souvent d'une violence extrême. La Provence a été le théâtre d'affrontements sanglants dès les premiers temps de la Révolution, la Terreur laissant place à une contre-révolution brutale qui s'essouffle sous le Consulat. Des « brigands » rouges ou blancs ont sillonné les routes, terrorisé les campagnes, mettant au défi l'ordre public dont l'État se proclame le garant. Parmi eux, le baron de Saint-Christol est dénoncé comme l'un des chefs royalistes les plus redoutables. Les autorités le dépeignent à la tête d'un réseau de brigands aussi cruels que déterminés ; elles le poursuivent avec acharnement sans parvenir à leurs fins. Saint-Christol est l'un des rares «brigands » de cette époque à avoir rédigé ses Mémoires, dans lesquels il présente de lui un tout autre portrait, celui d'un rebelle et avant tout d'un soldat du roi qui, n'ayant pas froid aux yeux, n'a reculé devant aucun danger pour faire triompher sa cause. Oscillant entre traité de philosophie politique et plaisir de raconter ses exploits, Saint-Christol livre, dans ses Mémoires, un point de vue unique sur le «brigandage » révolutionnaire dans la France méridionale.
\end{abstract}

Mots-clés : Contre-Révolution, brigandage, violences politiques, ordre public, justice, guerre civile, émigration, Provence. 
Parmi les figures du Midi contre-révolutionnaire, le baron de SaintChristol est un des rares «brigands » à avoir laissé ses mémoires ${ }^{1}$. Né à Tulette, à la frontière des États pontificaux et du Dauphiné, le 17 octobre 1748, Jacques François de Brémond, baron de Saint-Christol, était feudataire du Saint-Siège et assistait de droit aux assemblées des États du Comtat Venaissin. Le récit qu'il fait de sa vie retrace le parcours d'un rebelle. C'est au nom de sa patrie et de son honneur qu'il aurait pris les armes, poussé par des circonstances particulièrement difficiles au moment où, sous l'influence de la Révolution française, le Comtat s'apprête à devenir Français. La défense de la religion et de la monarchie aurait été le seul moteur de ses prises d'armes successives. Les autorités révolutionnaires présentent un tout autre portrait de lui, celui d'un « brigand » à la tête d'une bande d'hommes cruels et criminels. Le destin du baron de Saint-Christol bascule en 1790, alors qu'Avignon décide d'adopter la Constitution de la France et de demander son rattachement à cette nation toute neuve. Le Comtat Venaissin, qui relève aussi de l'autorité pontificale, forme une assemblée représentative, à la demande du baron de Sainte-Croix et de plusieurs autres nobles libéraux. Tout en affirmant son adhésion aux idées nouvelles, en contradiction avec l'opinion du Pape, l'assemblée s'attelle à une tâche complexe : sauvegarder les intérêts du Comtat tout en se gardant d'Avignon ${ }^{2}$, qui estime que son rôle est de se mettre à la tête de la Révolution dans les États du Pape afin de rompre avec des pratiques politiques jugées despotiques et archaïques ${ }^{3}$. L'imbroglio local devient rapidement un enjeu de la vie politique nationale. Chaque parti prend l'Assemblée nationale à témoin, ce qui permet à Robespierre de déclarer, le 16 novembre 1790 : «La cause d'Avignon est celle de l'univers, elle est celle de la liberté ». L'amalgame entre des Comtadins attachés à la religion et à l'autonomie du Comtat et les contre-révolutionnaires qui se manifestent en France est rapidement fait. Les débats se radicalisent et, tout comme Avignon s'était dotée d'une garde nationale patriote, l'Assemblée représentative se dote d'une milice. Les uns et les autres sillonnent le Comtat, afin de gagner les communautés à leurs vues : les premiers incidents éclatent en juillet 1790. Le baron de Saint-Christol se trouve engagé dès les premiers troubles,

(1) Précis des Mémoires de M. le baron de Saint-Christol, adjoint de l'agence royale de Souabe, depuis 1796 jusqu'en 1805, Avignon, Guichard, 1818, AD Vaucluse, Biblio. Art. 643 ; BNF, source Gallica, http://gallica.bnf.fr/ark:/12148/bpt6k46740d.

(2) Martine LAPIED, Le Comtat et la Révolution Française. Naissance des options politiques, Aix-en-Provence, Publications de l'Université de Provence, 1996 ; René MoulinAs, Histoire de la Révolution d'Avignon, Avignon, Aubanel, 1986, p. 68 et $s q$.

(3) Martine LAPIED, Le Comtat et la Révolution, op. cit., p. 63-70. 
l'assemblée représentative lui ayant confié le commandement des gardes nationales du Comtat. À ce titre, il participe aux expéditions destinées à s'opposer aux marches des gardes avignonnais et devient rapidement l'un des chefs de la Contre-Révolution aux yeux des patriotes provençaux.

Ses mémoires se présentent sous la forme d'un ouvrage imprimé de 88 pages, au format in- $8^{\circ}$, rédigés à la première personne. L'auteur se propose, dès les premières lignes, de se livrer à une analyse politique afin de rétablir la vérité sur une période trouble et sur ses propres engagements. "Aujourd'hui, libre sous la protection des lois, dégagé de toute considération, je vais montrer la vérité à ce peuple bon, sensible et humain $[. .]$.$» précise-il dans les toutes premières lignes. Le récit balance$ entre une dimension politique impersonnelle et la présentation parfois détaillée des péripéties auxquelles le confrontent la vie militaire et plus encore la clandestinité. Ces Mémoires ne sont pas la seule source dont l'on dispose pour saisir l'engagement du baron de Saint-Christol sous la Révolution, car les autorités locales l'on rapidement identifié comme l'un de leurs principaux ennemis, donnant à voir un personnage bien différent de celui campé par l'auteur des Mémoires ${ }^{4}$. L'écart se creuse au fur et à mesure que se déroule la Révolution : les autorités, concentrées sur sa traque, le dépeignent à la tête d'une bande nombreuse et puissamment armée, recrutant dans un espace méridional étendu au Comtat, au Vivarais, au Gévaudan et aux Alpes du sud. Rien de tout cela ne transparaît dans ses mémoires. Ce texte n'est ni un journal intime, ni le récit d'une vie, mais bien celui d'un engagement. Achevé un an à peine avant la mort de son auteur, il ne donne aucun détail sur son enfance, sa famille ou sa vie privée ; il s'ouvre sur la Révolution et s'achève avec la Restauration. S'il ne propose pas une vision originale de la Révolution, ni même une relation précise des faits, il permet une plongée dans l'univers mental d'un homme recherché comme l'un des principaux rebelles dans le Midi alors que ces derniers ne se sont guère racontés.

Nous avons entrepris de confronter ces différentes sources afin de dessiner le portrait d'un homme engagé dans une opposition frontale à la Révolution, vu par lui-même et par ses juges. Est-ce celui d'un « résistant », d'un « rebelle », d'un « brigand »? À travers le personnage du baron de Saint-Christol s'illustre la fragilité de la frontière qui sépare le contestataire du criminel. La rareté des écrits émanant de ceux qui ont été alors qualifiés

(4) AN, D/XXIV/1-3, papiers du comité d'Avignon, nommé le 17 juillet 1790 à la suite des troubles d'Avignon et du Comtat. 
de « brigands » fait toute la valeur des mémoires rédigés par le baron peu de temps avant sa mort. Nous nous attacherons tout d'abord à retracer l'entrée en résistance de Saint-Christol, selon ses écrits et d'après les documents d'archives. Nous tenterons ensuite de comprendre comment il a basculé dans une forme de rébellion plus violente en posant la question de son appartenance au brigandage qui instaure une forme de terreur dans le Midi provençal au lendemain de la chute de Robespierre.

\section{Le destin et l'honneur}

« Mon destin est d'encourager ceux qui sont entraînés par les circonstances, dans des affaires difficiles, à n'écouter que leur conscience, à ne prendre pour guide que l'honneur et à ne point pactiser avec le crime, afin qu'ils puissent dire Je n'ai rien à me reprocher : j'ai vu, malgré tous les obstacles, triompher mes principes, la Religion et la Monarchie $»^{5}$.

Les Mémoires du baron de Saint-Christol ne sont pas issus d'un journal tenu au moment des faits, mais ils sont clairement le fruit de sa volonté, au crépuscule d'une vie, de laisser un témoignage en guise de leçon pour l'avenir. Il reconnaît que des réformes étaient nécessaires dans les États pontificaux mais, près de trente ans après les faits, il dénonce sans nuance les divisions partisanes qui ont conduit, selon lui, aux excès et aux violences. De fait, celles-ci éclatent précocement à Avignon, où s'est établi de longue date un parti favorable au rattachement à la France ; les troubles gagnent rapidement le Comtat voisin, où les intérêts économiques divergent et l'attachement à la religion catholique reste ferme ${ }^{6}$. L'assemblée ordinaire des représentants des communautés comtadines de septembre 1789 obtient l'élection d'États généraux, qui se réunissent à la fin du mois de mai 1790 à Carpentras'. À cette date, le parti pro-français l'a emporté à Avignon. Des élections municipales ont conduit à la défaite des consuls en place sans mettre un terme à l'agitation partisane en ville. L'assemblée se trouve ainsi confrontée à une situation d'une très grande complexité. Le 11 juin, elle adopte la Constitution française, faisant de Pie VI un monarque

(5) Précis des Mémoires de M. Le baron de Saint-Christol, op. cit., p. 2 (en italiques dans le texte).

(6) Sur les questions de la formation des opinions politiques dans le Comtat, nous renvoyons à Martine LAPIED, Le Comtat et la Révolution Française, op. cit., chapitre II, p. 53-76.

(7) Les États généraux ne s'étaient pas réunis depuis 1596 ; en leur lieu et place, une assemblée générale se tenait chaque année pour régler les questions fiscales. Représentative des communautés, elle a contribué à établir l'autonomie administrative du Comtat. 
constitutionnel ; elle abolit les ordres et la féodalité et décrète les biens du clergé imposables. Mais dans le même temps, elle affirme sa fidélité au Pape ainsi que la volonté du Comtat à rester indépendant de la France. Consciente des difficultés que cette position va engendrer, elle se dote d'une garde nationale ou milice comtadine.

D'abord membre du Comité militaire de l'assemblée, le baron de Saint-Christol est nommé officier général des gardes nationales du $\mathrm{Comtat}^{8}$. Sa mission est de contrer par des manœuvres militaires l'action des Avignonnais. Des groupes de miliciens avaient en effet sillonné villes et bourgs pour remplacer les armes pontificales par celle de la France et diffuser une propagande favorable au rattachement à la France. Les expéditions militaires des Avignonnais sont perçues comme une réelle source de danger par les comtadins, mais ils sont peu nombreux à être déterminés à prendre les armes. L'assemblée elle-même se divise. Dans cet ensemble délétère, Saint-Christol représente le parti du Pape et de la fermeté ; il s'engage progressivement sur la voie d'une réaction marquée face à la position d'Avignon. En juin, des exécutions sommaires s'y étaient déroulées, conduisant à la mort plusieurs privilégiés et un taffetassier. Pour Saint-Christol, il ne s'agit de rien d'autre que de «brigandages », le marquis d'Aulan, l'une des victimes, ayant été spolié par l'un des partisans de la France, Lescuyer ${ }^{9}$. En juillet, il dirige une troupe de soldats sur la ville du Thor où des élections se déroulent dans un climat tendu. Proche d'Avignon, cette petite cité compte un parti pro-français qui appelle les Avignonnais à l'aide : la confrontation fait un mort et plusieurs blessés.

À la suite de ce premier engagement, Saint-Christol participe aux principaux affrontements qui se déroulent entre Avignon et le Comtat. Bien plus, confronté à la déliquescence de l'assemblée, il s'en proclame le président. En décembre 1790, il prend la tête d'une expédition contre les milices d'Avignon à Cavaillon. L'importance économique et politique de cette cité en faisait un enjeu dans la lutte d'influence entre Avignon et le Comtat. Cet affrontement a donné lieu à une littérature abondante et très partisane. Pour Saint-Christol, ce sont là « des brigands en nombre [qui] vinrent en armes, avec drapeaux, tambour et les armes de France. On fusilla, on fit des prisonniers [...] ». Saint-Christol se présente comme l'homme que les patriotes veulent abattre :

(8) Précis des Mémoires de M. le baron de Saint-Christol, op. cit., p. 12 ; Warren WILSON, Les réseaux contre-révolutionnaires en Provence sous la Révolution Française, Thèse de $3^{\mathrm{e}}$ cycle dactylographiée, sous la direction de Michel Vovelle, Aix, 1985, p. 38.

(9) Précis...op. cit., p. 11. 
« Général et président, je ne pouvois ignorer ma position, j’avois été averti de bonne part [...]. J'avois encouru la haine et la persécution des brigands de tous les pays. Je m'occupai du plan de défense : j'aurois inondé les campagnes, attendu dans les défilés les brigands, ayant de l'artillerie, de la cavalerie; et c'étoit ce que vouloient les braves paysans de Carpentras $\gg^{10}$.

C'est peu dire que les versions émanant des patriotes sont différentes. Ces derniers ont fait de ces affrontements le cœur d'un complot contrerévolutionnaire qui menacerait, depuis le Comtat, toute la Révolution Française. Les textes qu'ils produisent dans la presse locale ou à l'intention des députés de l'Assemblée nationale contrastent avec le récit de SaintChristol non seulement par leur contenu mais aussi par la profusion de détails sur l'affaire du Thor ou de Cavaillon, devenue l'emblème du martyre des patriotes locaux. Saint-Christol a conscience de l'inflexion du discours du parti pro-français. Il s'adresse aux autorités de la Drôme pour les engager à venir constater les violences exercées par l'armée d'Avignon et se présente comme un soldat au service des autorités civiles du Comtat, non comme un partisan ${ }^{11}$. Il ne fait aucun doute, à lire ses Mémoires, qu'il fallait préparer la guerre. C'est avec toutes les valeurs traditionnelles d'un noble combattant qu'il s'engage dans les combats, dont les Mémoires font un récit détaillé. Il est toujours dans la ligne politique que défend l'assemblée représentative du Comtat qui dénonce aussi les violences commises par les troupes d'Avignon et les pressions exercées pour influencer les communautés du Comtat en faveur du rattachement à la France. Les objectifs et le langage sont les mêmes : ils traduisent l'indignation des députés comtadins. À leurs yeux, les troupes avignonnaises se livrent à des brigandages, en dehors de tout respect de la légalité ${ }^{12}$.

Le début de l'hiver 1790-91 voit les tensions partisanes s'aggraver. Au début de janvier 1791, l'assemblée cesse de se réunir, alors que les troubles redoublent. Cavaillon est assiégée par les troupes venues d'Avignon; les combats de rue sont suivis du pillage des maisons de

(10) Précis, op. cit., p. 24-25.

(11) On constate un écart assez net entre le Précis des Mémoires de Saint-Christol (p. 20-21) et une copie d'un procès-verbal dressé par Saint-Christol lors de l'affaire de Cheval-Blanc, commune située à proximité de Cavaillon, que les troupes comtadines investissent le 25 décembre 1790 ; AD Drôme, L 184.

(12) Nouveau Manifeste des Représentants du Comtat Venaissin, 18 novembre 1790, AD Drôme, L 184 (Troubles du Comtat) ; p. 5, 6, 7 et 15, les troupes d'Avignon sont qualifiées de bandes ou hordes de «brigands ». Des extraits des registres municipaux de Cheval-Blanc et de Cavaillon, rédigés durant les troubles, utilisent le même vocabulaire. 
tous ceux qui étaient opposés au parti pro-français. On dénombre une quinzaine de morts et le double de blessés du côté des assiégés, sans compter les prisonniers emmenés dans les prisons d'Avignon. Le terme de «brigands » est désormais constamment utilisé pour désigner les troupes du parti avignonnais et pro-français. C'est en tant que président de l'assemblée représentative que le baron de Saint-Christol publie une adresse destinée aux députés de l'Assemblée nationale à Paris. Il y brosse un portrait sans concession des « hommes audacieux, des méchants [qui] se coalisèrent avec des traitres à la Patrie pour opérer la subversion de la Province. [...]

La ville d'Avignon, ou plutôt un ramas d'étrangers, de brigands qu'elle a dans son sein $[\ldots]$ portèrent un peuple fidèle et pacifique à la rébellion, à la trahison, au meurtre et au pillage [...]. De quel droit ces brigands et leurs complices peuvent-ils conquérir pour la France les États du Comté Venaissin $? »^{13}$.

Ce sont les désordres que dénonce le baron de Saint-Christol, et leurs cortèges de morts, d'injustices et de désolation dans les campagnes. L'armée avignonnaise se dirige alors vers Carpentras et campe sous ses murs. Après un échec en janvier, cette armée revient à la fin du mois d'avril. À cette date, un bon nombre de communautés du Comtat se sont ralliées à l'idée d'un rattachement à la France. Carpentras incarne, selon ses ennemis, le cœur du complot contre-révolutionnaire. Le siège dure un mois, pendant lequel les campagnes proches subissent les rigueurs de l'occupation militaire. Saint-Christol a gagné Paris où il essaie de convaincre les autorités et des hommes influents de la nécessité de respecter l'autonomie du Comtat. Sans relâche, il dénonce « l'anarchie et le brigandage qui désoloient le Comté Venaissin », en vain.

En mars en effet, trente-trois communautés du Comtat se sont fédérées dans une Union de Sainte-Cécile, contre l'avis de Saint-Christol, afin de résister aux projets d'Avignon ${ }^{14}$. La noblesse incarne désormais la voix de l'opposition, ce qui encourage les partisans de la réunion à la France à dénoncer un vaste complot dont les premières manifestations se seraient déroulées aux camps de Jalès (1790-1791). Des incidents éclatent à Vaison, où deux patriotes sont massacrés ${ }^{15}$. Avignon envoie des troupes, l'Union dépêche des hommes en armes : la bataille se déroule dans la plaine

(13) BM Avignon, ms 2989/13, p. 3 et 9.

(14) Martine LAPIED, Le Comtat et la Révolution, op. cit., p. 85-88.

(15) BM Avignon, ms 2989/34, diverses lettres concernant l'armée du Vaucluse ; AD Vaucluse, 9 J.7 (02). 
de Sarrians et tourne au désastre pour les fédérés, stimulant l'ardeur des gardes nationaux d'Avignon qui se concentrent, dès lors, sur Carpentras. Impuissant face à l'escalade, Saint-Christol rentre de Paris au lendemain de la défaite de l'Union ; il se rend à Villeneuve-lès-Avignon, où séjournent de nombreux exilés sortis d'Avignon, puis se décide à gagner Carpentras, qui subit la canonnade de l'armée de Vaucluse. Il est reçu par la municipalité de la ville ainsi que par les médiateurs désignés par la France à la fin du mois de mai 1791. Cependant, il tombe entre les mains de l'armée avignonnaise, parvient à s'échapper, apprend qu'il est recherché comme contre-révolutionnaire, ainsi que son fils. Le Comtat est alors le théâtre de scènes de pillage et de violences. L'armée de Vaucluse, dont le noyau est composé des gardes nationaux d'Avignon, pratique une politique de la terre brûlée autour de Carpentras, qui offre une étonnante résistance durant près de deux mois. Saint-Christol et son fils se résignent alors à l'exil.

\section{L'exil et la révolte}

Lorsqu'il se résout à quitter la France, le baron de Saint-Christol ne cache pas ses désaccords profonds avec les partisans de la Révolution. Il condamne comme traîtres à leur patrie les comtadins qui ont soutenu le projet de rattachement à la France mais aussi comme ennemis du roi de France, qu'il estime être retenu prisonnier. Les patriotes ne sont, à ses yeux, que des impies et des pillards. Mais sans doute cette position est-elle celle du vieil homme qui écrit ses mémoires plus de vingt ans après les événements. Lorsqu'il gagne Paris pour plaider en faveur de l'autonomie du Comtat, à la fin de l'hiver 1791, il n'affiche pas des idées aussi radicales. Il consulte beaucoup de monde, muni des recommandations de M. de Sainte-Croix, un temps figure de proue de la noblesse libérale du Comtat ${ }^{16}$. Mirabeau, Lafayette, le duc de Luynes, le député Bouche, élu par la sénéchaussée d'Aix, favorable à la réunion du Comtat à la France, entendent ses propos, promettent un soutien, interviennent au comité diplomatique. S'il choisit l'exil, comme le baron de Sainte-Croix, il n'imagine pas l'effondrement de tout ce en quoi il croit. Après une fuite rocambolesque, il parvient à Coblence. Parlant de lui à la troisième personne dans ce passage, il exprime son indignation mais ne résiste pas au plaisir de donner à voir ses aventures :

(16) Guillaume de Sainte-Croix (1746-1809), issu d'une noblesse ancienne, homme de lettres, était favorable à des réformes, ce qui l'avait conduit à être poursuivi par les autorités pontificales. En 1791, cependant, il est contraint à la fuite par l'armée des Avignonnais ; Antoine-Issac Sylvestre DE SACY, Notice sur M. Guilhem de Clermont Lodève de Sainte-Croix, Paris, Bibliothèque Royale, 1809. 
«Quel gouffre nous laissons derrière nous ! quel abîme se présente sur nos pas! Nous sortons de Grenoble au nombre de 25 ; on nous arrête, on nous attendoit pour nous pendre aux arbres du cours. Cette ville, jadis si polie, si honnête, n'étoit plus reconnaissable : nous aurions été assassinés sans la Garde Nationale qui nous jetoit dans les prisons pour nous garantir des mains des cannibales. Echappé à une mort qui paraissoit inévitable, je passe en Savoie [...]. Les paysans du Thouvet nous arrêtent : ils venoient de saccager le château de M. le comte de Marcieux. Le peuple souverain étoit tellement ivre qu'il prit une lettre de change pour un passeport, et nous laissa aller $\gg^{17}$.

Arrivé à Coblence, il s'engage dans la Légion Noire du vicomte de Mirabeau ; il fait également imprimer un texte, destiné aux princes, dont l'objectif est de fixer une ligne de conduite aux émigrés, qu'il juge trop divisés et trop hésitants : «Il fallait faire une guerre à mort, une chasse générale contre les loups de l'ordre social ${ }^{18}$. Par ces paroles, Saint-Christol se revendique comme un «ultra », de ceux qui, dans les armées des princes émigrés, veulent partir en croisade contre les jacobins. Il se serait ainsi radicalisé au cours de sa fuite hors du royaume. Joignant les actes à la parole, il participe à la campagne d'Alsace dans les rangs de l'armée de Condé, puis décide de rejoindre la France et sa fille qui, âgée de seize ans, est sans ressource et sans protection dans un pays troublé. Son retour n'est pas moins animé que sa fuite. À travers des lignes pittoresques, Saint-Christol offre de lui-même le portrait d'un homme plein de courage et d'énergie, qui n'a pas froid aux yeux. Arrêté en Alsace par un groupe de paysans, il est condamné à mort pour crime d'émigration, transféré dans la prison d'Huningue puis à Besançon, qu'il doit quitter pour subir sa peine dans la Drôme, dont la commune de Tulette dépendait. En chemin, il croise la route du maire de Strasbourg, Dietrich, emprisonné et condamné à mort, puis celle de fédérés de Lons-le Saulnier mis hors-la-loi pour avoir soutenu l'insurrection de Lyon à la fin juin $1793^{19}$. Enfin, approchant du terme de son voyage, il ne voit son salut que dans la fuite. Alors que son escorte traverse une forêt, que la route est déserte et ses deux gardiens sans méfiance, il passe à l'action :

« Je vois que l'instant est favorable pour mon évasion, personne alors ne se trouvant sur la route. Le courage double mes forces ; je lance une

(17) Précis..., op. cit., p. 39.

(18) Ibidem, p. 42.

(19) Jacques GoDeCHOT, La Contre-Révolution, 1789-1804, Paris, PUF, 1984 [1961], p. 255. 
poignée de tabac dans les yeux du gendarme qui conduisoit le char-à-banc, je saute à terre et je frappe le second gendarme au dos des jambes et sur la tête avec un bâton d'épineau que la geôlière de Besançon m'avoit donné pour me soutenir dans l'état de faiblesse que j'affectois. [...] Les deux gendarmes surpris et terrassés, je suivis l'aile du bois gagnant du côté de la Suisse. $[\ldots] »^{20}$.

Saint-Christol reprend le chemin de l'exil et de la guerre. La France dessinée par sa plume est tourmentée ; la confiance est ruinée, la force et la ruse sont les seuls recours dont dispose le fuyard. Après de nombreuses péripéties, il retrouve enfin les troupes de Condé et reçoit le commandement d'une compagnie. Blessé en décembre 1793, il ne peut rester sur le front et trouve refuge en Suisse. Plongé dans l'incertitude la plus complète, son destin bascule au gré des rencontres que le hasard lui ménage. La vie dans la clandestinité lui fait rencontrer d'autres opposants à la Révolution, qui hantent les frontières agitées par les armées des princes émigrés. C'est ainsi qu'il croise le chemin des fédéralistes rencontrés à Lons-le-Saunier et s'engage dans un complot visant à fomenter une insurrection armée. Le secret éventé, il est sommé de quitter la Suisse. Il gagne l'Italie, où séjourne aussi le comte de Provence, avant de rejoindre le prince de Condé à Baden-Baden. Il se partage alors entre des missions secrètes et les combats, ce dont les Mémoires rendent compte de manière parfois confuse.

La chute de Robespierre lui permet de rentrer en France mais, n'écartant pas complètement la perspective d'une rébellion armée, il entre en contact avec les réseaux contre-révolutionnaires provençaux, sur lesquels il donne peu de détails. La situation locale était très complexe : aux côtés des canaux royalistes « contrôlés » par Louis XVIII, Condé ou les agents Anglais, on trouvait tout un ensemble de chefs locaux, à la tête de bandes armées, que les princes et leurs correspondants ne maîtrisaient pas toujours. La noblesse locale avait constitué un premier réseau autour de grands aristocrates exilés dans le comté de Nice et à Gênes, autour du marquis de Marignane, beau-père de Mirabeau, et du fils d'un parlementaire aixois, Vernègues. Ils sont rejoints par d'autres opposants comme Monnier de la Quarrée, fils d'un notaire de Viens (Basses-Alpes) ou encore le marquis de Bésignan, issu de la noblesse seconde du bas Dauphiné, tout comme Saint-Christol $^{21}$. Ces réseaux étaient en contact avec l'agent anglais Francis

(20) Précis..., op. cit., p. 46-47.

(21) Warren WILSON, Les réseaux contre-révolutionnaires en Provence sous la Révolution Française, op. cit., p. 20-21, p. 61-69, p. 92. 
Drake, installé à Gênes. La fin de la Terreur ravive les projets de contrerévolution armée en Provence. Les Jacobins sont divisés et le soutien populaire sur lequel ils s'appuyaient dans de nombreuses villes mais aussi dans les campagnes, est fragilisé par la crise économique tout autant que par le déchaînement de violences auquel la Terreur a donné lieu ${ }^{22}$. Enfin, le retour progressif des émigrés ainsi que de nombreux déserteurs au début de 1795, contribue à la dégradation de la vie politique. Moins d'une année après son retour, Saint-Christol s'engage dans la voie d'une réaction armée. Il entre en contact avec Job Aymé, membre du réseau contre-révolutionnaire provençal, ancien procureur syndic général de la Drôme (en 1792) et avocat de Saint-Christol. L'agitation se propage dans le Midi : des bandes armées s'attaquent aux représentants des autorités dans le Gard, la Lozère, le Vaucluse, les Basses-Alpes et les Bouches-du-Rhône. En Haute-Loire, un rassemblement de plusieurs milliers d'hommes dirigés par le chevalier Lamothe, marche sur Yssingeaux ${ }^{23}$. À ses côtés figure Dominique Allier, frère de l'un des chefs des camps de Jalès, soldat redoutable qui s'était mis à la tête d'une troupe d'une centaine d'hommes. Obéissant à une discipline toute militaire, ils terrorisaient les patriotes de la Lozère, de l'Ardèche et de la Haute-Loire ${ }^{24}$.

Alors que la poussée des royalistes en Provence se confirme, que des insurrections éclatent dans plusieurs départements, notamment à l'Ouest, l'heure semble avoir sonné pour une nouvelle insurrection armée aux yeux de Saint-Christol : « Tout annonçoit que l'instant étoit favorable pour prendre les armes et secouer le joug de la plus honteuse tyrannie. [...] Je lève l'étendard de la guerre par une Proclamation propre à exciter le courage et l'enthousiasme des Royalistes. ${ }^{25} \gg$ Cette proclamation prend la forme d'un «Manifeste du commandant en chef de l'armée du Corps

(22) Un tribunal révolutionnaire a été créé à Orange pour juger les coupables de contrerévolution de la région. À Bédoin, village situé au pied du Ventoux, arbre de la liberté est arraché au cours de la nuit du $1^{\mathrm{er}}$ mai 1794. 63 prisonniers sont condamnés à mort et exécutés ; le village est vidé de ses habitants, ses terres partagées entre les communes voisines et les maisons incendiées. Cet épisode est l'un des plus traumatisants de l'histoire de la Révolution en haute Provence. Je renvoie notamment à Martine LAPIED, Le comtat et la Révolution Française, op. cit., chapitre VI ; Pierre Charpenne, Les grands épisodes de la Révolution dans Avignon et le Comtat, Avignon, impr. H. Guigou, 1901, 4 vol., T. III, p. 126-186.

(23) AN, Police générale, F/7/3681/5 (Haute-Loire) et BB/18/439 \& 440 ; voir aussi Gilles ChARreyron, Politique et religion. Protestants et catholiques de la Haute-Loire, Clermont-Ferrand, Institut d'Études du Massif central, 1990, p. 239-242 ; Lamothe, ancien soldat, est, tout comme Bésignan, une «tête brûlée » de la contre-révolution méridionale.

(24) Valérie SOTTOCASA, Mémoires affrontées. Protestants et catholiques face à la Révolution dans le sud du Massif-Central, Rennes, PUR, 2004, p. 179.

(25) Précis..., op. cit., p. 58. 
législatif aux habitants du Midi $»^{26}$ à travers lequel Saint-Christol s'adresse aux « citoyens » endeuillés par les « entreprises des brigands [...] de ces monstres insatiables, encore altérés du peu de sang que leur longue tyrannie vous a laissé $[. .$.$] ». L'auteur les encourage à se «rallier à la voix des$ Pichegru, des Willot $\gg^{27}$ en rejoignant, sous les murs d'Orange, le général en chef qui écrasera «cette infâme commune » où fut réuni le Tribunal révolutionnaire pendant la Terreur. L'objectif de cette prise d'armes semble donc de coordonner les efforts des royalistes de l'intérieur et de l'extérieur pour faciliter le renversement du gouvernement. Les Mémoires de SaintChristol laissent entendre qu'il agit dans le cadre d'une vaste réaction royaliste et la concomitance de divers soulèvements avec l'attaque anglaise à Quiberon semble accréditer cette thèse. En réalité, l'auteur reste très vague sur ses soutiens : «en passant à Lyon, des braves officiers et soldats de l'armée de Condé promirent de me joindre partout où je parviendrois à former un rassemblement $[\ldots] »^{28}$. Son objectif est d'entraîner une vaste insurrection dans la région, de prendre des armes dans la citadelle de Pont-Saint-Esprit, d'écraser Orange et Avignon, symboles du jacobinisme local, et de favoriser ainsi la circulation des troupes royales censées entrer en France derrière Condé.

Les premiers pas des rebelles à la fin de fructidor an $\mathrm{V}$ (mi septembre 1797), sont couronnés de succès. La cité de Pont-Saint-Esprit se rend, la citadelle est contrôlée après quelques affrontements mineurs. Saint-Christol se saisit des armes et s'apprête à prendre la direction d'Orange lorsqu'il apprend le coup d'État du 18 fructidor (4 septembre 1797). Sa troupe se débande alors très vite et abandonne la citadelle à peine conquise (28 fructidor/14 septembre 1797), craignant un retour de la Terreur. SaintChristol tente de gagner la montagne pour y former, dit-il, « une seconde Vendée $»^{29}$. Il n'en n'a pas le temps : à peine a-t-il regagné sa paroisse natale de Tulette qu'il est fait prisonnier par les autorités de sa commune et enfermé au château. Il parvient à s'en échapper quelques heures plus tard, grâce à la complicité de ses hommes. Mais, après avoir détruit les munitions enlevées à Pont-Saint-Esprit, et sachant sa vie menacée, il se résout à reprendre le chemin de l'exil, en direction de la Savoie et de

(26) Pierre Charpenne, Les grands épisodes de la Révolution, op. cit., T. IV, p. 201-202.

(27) Ces deux généraux étaient acquis aux royalistes, plutôt du côté des modérés, les « clichyens », et avaient été élus députés lors des élections de l'an IV. Les royalistes sont encore très divisés sur la question du régime à restaurer et sur les moyens d'y parvenir ; voir Jacques GODECHOT, La Contre-Révolution, op. cit., p. 298-310.

(28) Précis..., op. cit., p. 57.

(29) Ibidem, p. 60. 
l'armée du Prince de Condé. Les autorités ne parlent plus de lui que comme du chef d'une bande de brigands, ce qu'il nie dans ses Mémoires, qui sont néanmoins très vagues sur la suite de son implication dans la contre-révolution provençale, plus encore sur ses liens avec les bandes qui participent activement à la Terreur blanche dans la région.

\section{Du rebelle au brigand : la part d'ombre du baron de Saint-Christol}

Le récit du baron de Saint-Christol se concentre sur les conditions de sa fuite, avec le sens des rebondissements qui caractérise l'écriture des Mémoires. Son retour à la clandestinité le conduit à renouer avec des habitudes de vie qui sont celles des marginaux ou des criminels. Caché par son fermier avec les hommes qui l'ont délivré, il ne peut rester trop longtemps dans la place. Les fuyards se séparent et Saint-Christol prend seul la route de l'exil. Il se réfugie dans les maisons qui acceptent de l'abriter, talonné par les soldats de la République. Durant tout un mois, il est caché dans une falaise où on lui ménage « une niche sous un rocher »; certains élus protègent sa fuite, au nom de solidarités anciennes ou politiques. Il gagne Grenoble et traverse les montagnes pour rejoindre les armées de Condé, échappant à deux reprises à des avalanches avant d'atteindre le Valais. Après l'invasion de la Suisse, il rejoint l'agence royale de Souabe au cours de l'hiver $1797^{30}$. Il prend part aux réunions les plus importantes où il côtoie les princes, les émissaires des Anglais ou les représentants de l'Empereur d'Autriche. C'est en cherchant à rejoindre l'Angleterre que son fils, militaire et royaliste engagé comme lui, est arrêté en France, traduit dans la prison du Temple, condamné à mort et exécuté. Le père endeuillé s'attarde peu sur ses sentiments : «Enseveli dans une retraite profonde, je pleurois mon fils dans le silence de la douleur [...] Lorsque je fus réveillé par le bruit de l'arrestation d'un individu [...] » qu'il pense, à juste titre, être le duc d'Enghien. Aussitôt, il reprend du service auprès de Condé ${ }^{31}$. De

(30) Cette agence appartient aux réseaux contre-révolutionnaires de renseignements; le premier a été celui que le comte d'Antraigues a mis sur pied, la « Manufacture », découvert et détruit en 1797 ; des 1798, les royalistes créent l'Agence de Souabe, organisée autour du comte de Précy, que Saint-Christol avait rencontré lors d'une mission peu de temps avant son retour en France, des députés Dandré et Imbert-Colomès, du général Willot, chargé de la 8 e division militaire couvrant en grande partie la Provence septentrionale. L'agence avait pour mission de ranimer la Contre-Révolution en France, notamment autour des Instituts Philanthropiques ; voir Jaques GODECHOT, La Contre-Révolution, op. cit., p. 361-364.

(31) Louis Antoine Henri de Bourbon Condé, duc d'Enghien, était le petit-fils du prince de Condé et servait dans son armée ; Bonaparte le fait enlever à Ettentheim puis exécuter après un simulacre de procès le 21 mars 1804 ; Dictionnaire de la Contre-Révolution, Jean-Clément MARTIN (dir.), Paris, Perrin, 2011. 
nouveau traqué, il fuit à Munich où il apprend la proclamation de l'Empire, qu'il condamne avec vigueur. Cependant, sans doute par prudence, SaintChristol passe très vite sur la décennie qui s'écoule. Il achève ses Mémoires avec la peinture de sa vieillesse, éclairée par le bonheur d'avoir vu ses idéaux triompher :

«Calme après la plus violente tempête, la France a retrouvé sous ses Rois la paix et le bonheur. Le passé nous a prouvé d'une manière trop cruelle que la désolation est le partage des Royaumes que la discorde et les factions déchirent et tentent de renverser. Après le sort funeste de cette multitude d'ambitieux jaloux du souverain pouvoir, qui n'ont fait que passer se culbutant les uns sur les autres, quel mortel insensé oseroit encore porter le trouble dans sa patrie ? $»^{32}$.

Cependant, c'est bien comme factieux criminel que les autorités révolutionnaires l'ont fait rechercher, comme chef d'une bande de brigands dans le Vaucluse, la Drôme et l'Ardèche. L'écart entre les Mémoires et les sources d'archives est flagrant. Les autorités patriotes auraient-elles inventé un personnage, construit un brigand fictif afin de stigmatiser l'opposition royaliste armée? C'est au lendemain de la prise de Pont-Saint-Esprit que les autorités dénoncent en lui le "général en chef de cette armée de brigands $»^{33}$. Elles ordonnent que les municipalités prennent des mesures pour que soit organisée une battue «à l'effet de s'assurer de tous les brigands qui y seront découverts ». Pourquoi qualifier ces hommes de «brigands »? Il est un fait que depuis l'insurrection de la Vendée, les autorités révolutionnaires ont pris l'habitude de désigner ainsi les chouans et vendéens révoltés ${ }^{34}$. En Provence, l'amalgame entre des bandes violentes et politisées et les brigands remonte aux premiers heurts politiques, comme nous avons pu le lire sous la plume de Saint-Christol. L'insurrection de la Vendée oriente l'usage du terme : le brigandage est désormais le fait des contre-révolutionnaires et non plus des patriotes. Si la Provence n'a pas donné naissance à une insurrection comparable à celle de la Vendée, elle est le théâtre d'une Terreur blanche d'une très grande violence en 1795. Aux exécutions de la Terreur jacobine répondent les assassinats de

(32) Précis..., op. cit., p. 87-88.

(33) AN, F/7/3652/2, extrait du procès verbal des séances de l'administration centrale du département de l'Ardèche, $3^{\text {e }}$ jour complémentaire an V (14 septembre 1796).

(34) En particulier depuis le rapport de Bertrand Barère, Rapport sur la Vendée au nom du Comité de salut public, Assemblée nationale, séance du $1^{\mathrm{er}}$ octobre 1793, source Gallica, http://gallica. bnf.fr/ark:/12148/bpt6k787546. 
patriotes et d'élus. Les modalités des deux épisodes de Terreur ne sont pas identiques, la première usant de l'appareil judiciaire, l'autre d'une forme de criminalité au service de la politique. Les élus subissent des pressions, les juges sont victimes d'attentats, les témoins n'osent plus franchir la porte des tribunaux, des communautés rurales tout entières se murent dans le silence. Traquer ces bandes relève à la fois d'une position idéologique - la lutte contre les royalistes - et d'une stratégie de survie pour les membres des administrations qui, par leur patriotisme, se savent menacés de mort.

Saint-Christol se comporte-t-il en «brigand »? Tout comme eux, il se met à la tête d'un rassemblement, une « armée » pour l'auteur des Mémoires qui, à la suite de sa déroute, se disloque en plusieurs bandes, selon les autorités. Elles auraient à leur tête des « lieutenants » de SaintChristol et se livreraient à une chasse à l'homme sans pitié contre les élus et les patriotes. De fait, les assassinats se multiplient dans la région où cette armée se serait dispersée, à la frontière entre le Vaucluse, l'Ardèche et la Drôme. Le jour même de sa dispersion, elle aurait fusillé plusieurs personnes et commis des vols en traversant la ville de Bollène ${ }^{35}$. Quant à l'implication de son chef dans la vague d'assassinats qui suit, les sources émanant des autorités militaires, civiles ou judiciaires restent assez vagues, dénonçant plutôt « l'armée de Saint-Christol » ou ses principaux lieutenants. L'un d'entre eux, Montauban, sème la terreur dans la partie septentrionale du Vaucluse longtemps après l'affaire de Pont-Saint-Esprit. À la tête d'une troupe de cent cinquante hommes armés, il pille les domaines des républicains, arrête les malles et les courriers, commet ses crimes au nom du Christ ${ }^{36}$. Saint-Christol l'aurait rencontré quelques temps avant le rassemblement du 20 fructidor, ainsi que d'autres têtes brûlées de la contre-révolution provençale, notamment un certain Pastour, qui terrorisait la région de L'Isle-sur-Sorgues. L'accusateur public du tribunal criminel affirme, pour sa part, que la bande de Saint-Christol aurait formé une société secrète, appelée les « chevaliers de la lune », dont les membres porteraient un ruban noir et une ganse blanche. Aux yeux des autorités, ces hommes sont des monstres : « De tout ce que le brigandage poussé à l'excès peut avoir de plus scélérat, un monstre appelé Montauban, mannequin de gens mal intentionnés et ennemis de la République [...] en une seule décade [ils] ont pendu un républicain, le nommé Caderousse, ils en ont noyé deux

(35) AN, F/7/3652/2.

(36) AN, BB/18/888B, Lettre de la municipalité d'Orange au ministre de la Justice, 3 messidor an $\mathrm{V}$ (21 juin 1797). 
autres et ont fini la décade par le meurtre d'une jeune républicaine $[\ldots] \gg^{37}$. Quant à la bande de Pastour, elle n'est pas moins terrible : son chef passe pour couper les oreilles ou le nez de ses victimes et les abattre au cours de certaines parties de cartes ${ }^{38}$. Pastour, âgé de vingt-quatre ans, aurait été arrêté en ayant quatorze nez humains dans ses poches! Les autorités l'accusent clairement d'avoir pris part à l'insurrection de Saint-Christol ; il aurait ensuite trouvé refuge dans la région de Pertuis et se serait caché dans le sud du département des Basses-Alpes, où ses hommes se seraient livrés à des égorgements « en masse $»^{39}$.

Le bras droit de Saint-Christol serait Dominique Allier. Natif du Gévaudan, impliqué dans les camps de Jalès, dans l'insurrection du Gévaudan en mai 1793 et aux côtés de Lamothe en Haute-Loire en 1797, il aurait rencontré le baron de Saint-Christol dans le plus grand secret peu de temps avant l'attaque contre la citadelle de Pont-Saint-Esprit. Leur association est attestée par le témoignage de plusieurs personnes accusées d'avoir pris part à ce rassemblement, ainsi que par le jugement de la commission militaire de Lyon, qui le condamne à mort pour en avoir été l'un des chefs ${ }^{40}$. Un rapport signé du ministre de la Guerre sur l'agitation royaliste dans le Midi, évoque en ces termes la complicité entre Allier et Saint-Christol : «La prise momentanée du fort Saint-Esprit par 400 brigands, à la tête desquels était Dominique Allier, cet éternel conjuré, et Saint-Christol, se qualifiant de Général en chef de l'armée des Deux Conseils $»^{4}$. Saint-Christol ne mentionne pas cette complicité mais insiste sur la coordination entre sa prise d'armes et les projets contrerévolutionnaires des émigrés. Cependant, dans la réalité, la coordination entre les rebelles de l'intérieur et les forces émigrées est moins évidente. Les insurrections dans le Midi se réduisent à des coups de main qui échouent systématiquement, depuis les premiers camps de Jalès (1790-92) jusqu'à la prise d'armes de Saint-Christol en l'an V, faute de discipline. Dans l'entourage de Louis XVIII ou du prince de Condé, les avis portés sur les rebelles du Midi sont plus que réservés.

(37) Ibidem.

(38) AN, BB/18/888/A, lettre des républicains avignonnais, des républicains de Sorgues, de messidor et fructidor an $\mathrm{V}$ (juin et juillet 1797).

(39) AN, BB/18/888/A, lettre du 22 vendémiaire an VI (13 octobre 1797) ; AD Vaucluse, 7 $\mathrm{L}$ 95, liste des assassinats commis dans la région de Pertuis.

(40) AN, BB/18/124, déposition du 16 ventôse VI (6 mars 1798) ; BB/18/125, jugement du 25 brumaire VII (15 novembre 1798).

(41) AN, AF/III/44, dossier 159. 
Sur le terrain cependant, ils se sont révélés de redoutables combattants. Harcelant les patriotes et les autorités révolutionnaires, ils ont su instaurer un désordre tel que l'État a été mis en échec dans ses missions d'ordre et de sécurité durant plusieurs années. Vivant en marge de la société, aux dépens de la solidarité des populations ou d'une partie d'entre elles, ils ont su tirer un trait sur le confort de leur existence antérieure à la Révolution. Ces hommes sont en effet presque toujours issus d'une élite sociale composée à la fois de représentants de la noblesse seconde et de la bourgeoisie locale. Les bandes recrutent au sein de toute la société, parmi les petits notables, les artisans, les ouvriers et les paysans, plus modestement. Le gros des troupes est formé de déserteurs et de réquisitionnaires insoumis. Cette jeunesse rurale permet d'ancrer la résistance royaliste dans les villages, sur la base d'une solidarité familiale et de voisinage que la pression exercée par les bandes royalistes et leur brutalité renforce clairement. Saint-Christol ne donne aucun détail sur ses contacts avec les chefs des bandes royalistes du Midi, mais trop de témoignages convergent pour laisser croire qu'il a agi seul. La criminalisation de l'opposition politique a incontestablement poussé les royalistes engagés dans la résistance militaire à se lier avec des hommes que rien n'aurait amené à se fréquenter avant la Révolution. Ils ont également été conduits à adopter une forme de combat plus proche de la guérilla que de la guerre traditionnelle, adoptant parfois des comportements d'une extrême violence. Saint-Christol n'aborde pas du tout cet aspect des combats royalistes, ce que l'on peut comprendre compte tenu du fait que ses Mémoires ont été rédigés bien après les faits, alors qu'il était devenu un vieil homme respectable, retiré dans le petit bourg de Beaumes-de-Venise. Mais comment aurait-il pu ignorer ces pratiques, qui étaient monnaie courante au sein même des troupes qu'il a levées et menées au combat ? D'ailleurs, des exécutions ont eu lieu lorsque ses troupes ont abandonné Pont-Saint-Esprit (sept ou quinze morts à Bollène ?) et des maisons ont été pillées. Dans les semaines qui suivent, les violences ne cessent de s'accroître. La traque s'accentue, malgré le caractère lacunaire et imprécis des informations détenues par les autorités : celles du Vaucluse savent que Saint-Christol s'est retrouvé seul et s'est enfui mais en Ardèche on pense qu'il est de nouveau aux côtés de Dominique Allier. Les battues permettent de nombreuses arrestations : Lamothe, au printemps 1797 et Dominique Allier en juillet de l'année suivante. Saint-Christol a sans doute regagné la cour des princes émigrés à cette date, comme il l'affirme dans ses Mémoires. Les renseignements qui le concernent dans les archives de police et de justice sont de plus en plus flous ; son nom cesse d'y apparaître au cours de 1799. 
Figure de l'opposition à la Révolution, le baron de Saint-Christol est peu à peu devenu une incarnation du brigandage contre-révolutionnaire dans le Midi provençal. Les contemporains et les historiens ont souligné le caractère à la fois précoce et brutal des violences méridionales, les attribuant au tempérament des hommes du sud, au caractère implacable du soleil, ou encore à un cycle séculaire de vengeances claniques. Ce n'est pas l'objet de cette contribution que de discuter ces positions, que nous ne partageons pas, mais bien d'observer les affrontements provençaux à travers les écrits de l'un de ses acteurs. Ses Mémoires sont ceux d'un rebelle, d'un résistant et d'un soldat, pas d'un «brigand », sur le mode qui sera adopté par Vidocq dans ses Mémoires ${ }^{42}$, car Saint-Christol n'assume pas cette image. Peut-on même penser que son texte a pour enjeu de laver son nom de tout soupçon de brigandage ? La vision qu'il donne de lui-même diffère assez nettement de celle qui se dessine sous la plume des représentants des autorités révolutionnaires. S'agit-il seulement d'une part d'ombre que le baron refuse d'assumer plus de vingt ans après les faits ? Sans doute cette dimension est-elle incontestable car si l'objet avoué du récit est de montrer la fierté d'avoir su se dévouer avec courage et honneur à des idéaux, les moyens utilisés étaient plus difficiles à avouer. Prolixe quand il s'agit de décrire ses aventures lorsqu'il fuit sur les chemins de l'exil, Saint-Christol reste très vague lorsqu'il aborde les conditions dans lesquelles il a mis sur pied l'insurrection de l'été 1797, refusant de citer le nom de ceux qui y ont collaboré. En 1814, certains acteurs des soulèvements royalistes provençaux étaient toujours en vie. Pour leur part, les autorités dénoncent des complicités avec des hommes qui ne reculaient ni devant les pillages, ni devant les assassinats pour terroriser les patriotes. Certes, menacés de mort, ces hommes avaient tendance à voir partout des complots. Mais dans ce cas, il ne nous semble pas possible que Saint-Christol ait pu se passer de l'appui logistique et humain des bandes royalistes implantées dans la région, d'autant qu'il était resté absent plusieurs années durant. Que pouvait-il savoir exactement de leurs comportements ? Sans doute lui avait-on rapporté les hauts faits d'un Dominique Allier, qui soignait la mise en scène de ses interventions. Il ne pouvait pas ignorer les agissements du marquis de Bésignan, ou du chevalier de Lamothe. Du même coup, pouvait-il ignorer qu'à la cour des princes émigrés, les avis étaient très réservés sur leurs coups de mains ? Cela dut le conduire à traiter avec Allier,

(42) Mémoires de Vidocq, chef de la Police de sureté jusqu'en 1827, Paris, Tenon, 1828, source Gallica, http://gallica.bnf.fr/ark:/12148/bpt6k10407102. 
Lamothe et consorts dans le plus grand secret et explique peut-être qu'il ait pu retrouver une place auprès de Condé dès son retour en exil après l'échec de l'insurrection de l'an $\mathrm{V}$; ou encore qu'il ait obtenu une pension de Louis XVIII au titre de sa carrière militaire dans l'armée de Condé.

Du côté de l'état major émigré, il semble que l'on ait fait la différence entre le baron de Saint-Christol et les brigands royaux, mais pas du côté des révolutionnaires. À leurs yeux, Saint-Christol est le chef d'une bande de brigands, qui pille, vole et tue. Au temps de la Terreur blanche, la guerre civile n'est pas une guerre en dentelle. De part et d'autre, les violences sont extrêmes. Saint-Christol ne pouvait l'ignorer quand il a approché des hommes comme Montauban, Pastour ou Allier. Peu de choses semblent séparer les royalistes armés et les bandits de grand chemin : tous représentent un défi pour le maintien de l'ordre et de la confiance, indispensables pour assurer la stabilité du régime républicain. Or, dans le Midi, cette sécurité n'existe pas. Les routes ne sont pas sûres, les bastides isolées sont pillées par des bandes armées, les caisses publiques sont volées. L'amalgame entre les brigands de droit commun et les bandes royalistes est autant le fruit d'une manipulation de l'opinion que celui de la peur inspirée par la vague de violences de la Terreur blanche. Saint-Christol devient alors l'illustration de la perversion de l'opposition royaliste, tant du fait de ses origines nobles ou de son attachement à la monarchie et à l'Église catholique romaine, que de ses relations avec les bandes royalistes engagées dans une guérilla violente. Son exemple illustre la difficulté rencontrée par les opposants politiques durant la période révolutionnaire : contre-révolutionnaires, ils devenaient une menace pour la patrie et devaient être éliminés. Rejetés dans la clandestinité, ils étaient parfois amenés à fréquenter des délinquants ou à adopter certains de leurs comportements, devenant, sous la plume des autorités, des « brigands ». Rebelles en armes ou brigands, la frontière est très difficile à tracer entre les bandes royalistes et les bandits de droit commun ${ }^{43}$. La lecture des Mémoires du baron de

(43) À titre de comparaison entre le Midi et les territoires belges annexés, voir Xavier ROUSSEAUX, « Rebelles ou brigands ? La guerre des paysans dans les départements "belges" (octobredécembre 1798) », Cahiers d'histoire. Revue d'histoire critique, $\mathrm{n}^{\circ}$ 94-95/2005, p. 101-132. 
Saint-Christol et des archives de l'époque révolutionnaire offre une rare occasion de confronter des voix toujours discordantes.

Valérie SOTTOCASA

Université de Toulouse-II Le Mirail

Framespa, UMR5136

Valerie.sottocasa@wanadoo.fr 